\title{
BMJ Open Is body mass index associated with symptom severity and health-related quality of life in irritable bowel syndrome? A cross-sectional study
}

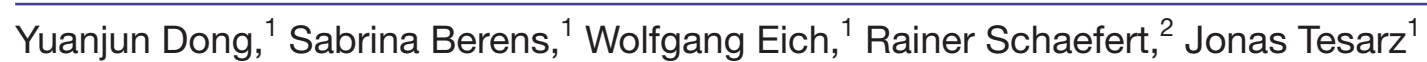

To cite: Dong Y, Berens S, Eich $\mathrm{W}$, et al. Is body mass index associated with symptom severity and health-related quality of life in irritable bowel syndrome? A crosssectional study. BMJ Open 2018;8:e019453. doi:10.1136/ bmjopen-2017-019453

- Prepublication history for this paper is available online. To view these files, please visit the journal online (http://dx.doi. org/10.1136/bmjopen-2017019453).

Received 5 September 2017 Revised 10 August 2018 Accepted 16 August 2018

Check for updates

(c) Author(s) (or their employer(s)) 2018. Re-use permitted under CC BY-NC. No commercial re-use. See rights and permissions. Published by BMJ.

${ }^{1}$ Department of General Internal Medicine and Psychosomatics, University Hospital Heidelberg, Heidelberg, Germany ${ }^{2}$ Department of Psychosomatics, University Hospital Basel, University of Basel, Basel, Switzerland

Correspondence to Dr Jonas Tesarz; Jonas.Tesarz@med.uniheidelberg.de

\section{ABSTRACT}

Objective The aim of this study was to describe the body mass index (BMI) distribution in patients with irritable bowel syndrome (IBS) based on the Rome III criteria and to evaluate the association of BMI with symptom severity and quality of life (QOL).

Methods A cross-sectional study was carried out in patients visiting our outpatient functional gastrointestinal disorders specialty clinic. IBS diagnosis was made based on Rome III criteria. IBS symptom severity was investigated using the IBS severity score system. QOL was assessed using the Short Form 36 Health Survey, which consists of physical health and mental health.

Results 366 patients (252 women) who fulfilled Rome III criteria and provided complete BMI data $(23.90 \pm 5.22 \mathrm{~kg} /$ $\mathrm{m}^{2}$ ) were included. Overall, $59.0 \%$ of patients with IBS were in the normal weight range, $30.3 \%$ were overweight or obese, and $10.7 \%$ were underweight. Both physical and mental health decreased significantly with the severity of symptoms (all $p<0.01$ ), while controlling for several covariates (age, gender, family status, education status and IBS subtypes). Obesity and symptom severity $\left(\beta=-0.177, \triangle R^{2}=0.037, p<0.01 ; \beta=-0.387, \Delta R^{2}=0.147\right.$, $p<0.01)$ were significant negative factors that influencing physical health. Symptom severity $\left(\beta=-0.301, \triangle R^{2}=0.084\right.$, $\mathrm{p}<0.01$ ) was significant negative factor that influencing mental health. However, BMI didn't account for additional variance in mental health $(p>0.05)$.

Conclusion Being overweight is a common phenomenon in patients with IBS regardless of IBS subtype. The association between QOL and symptom severity followed a negative dose-response pattern. Patients with higher BMI, especially obese patients, were more frequently in poor physical health. However, this kind of relationship was not found in BMl and mental health.

\section{INTRODUCTION}

Irritable bowel syndrome (IBS) is a chronic and common functional bowel disorder that is characterised by recurrent abdominal pain or discomfort associated with altered bowel habits. ${ }^{1}$ According to the Rome III criteria, IBS is classified into four subtypes (IBS with diarrhoea (IBS-D), IBS with constipation (IBSC), mixed IBS (IBS-M) and unsubtyped IBS

\section{Strengths and limitations of this study}

- This study uses a large and well-described patient cohort with a validated diagnosis of irritable bowel syndrome (IBS) based on the Rome III criteria.

- The association between quality of life (QOL) and symptom severity followed a negative dose-response pattern.

- Patients with higher body mass index (BMI) were more frequently in poor physical health. However, this kind of relationship was not found in BMI and mental health of QOL.

- The study was cross-sectional, therefore it is not possible to infer causation.

- BMI cannot fully reflect the impact of the factors of fatty mass, dietary habit, other lifestyle factors such as physical exercise on IBS.

(IBS-U)) based on the predominant abdominal symptomatology. ${ }^{1}$ It has been frequently reported that IBS leads to impaired quality of life (QOL).$^{2-4}$ The symptoms of IBS are associated with different eating habits. ${ }^{5}$ In a recent randomised controlled trial, a diet low in fermentable oligosaccharides, disaccharides, monosaccharides and polyols was shown to improve the IBS-D patients' QOL, anxiety and activity impairment. ${ }^{6}$

Epidemiological studies have shown that as many as $16.5 \%$ of adults in European countries are obese (body mass index (BMI) $\left.>30 \mathrm{~kg} / \mathrm{m}^{2}\right)^{7}$, and that the incidence of obesity is increasing. ${ }^{8}$ Additionally, a cross-sectional multicentre study found that more than $63 \%$ of outpatients and $80 \%$ of inpatients in gastroenterological centres suffered from significant changes in body composition. ${ }^{9}$ In addition, underweight (BMI $<18.5 \mathrm{~kg} / \mathrm{m}^{2}$ ) or obese body condition (BMI $>30 \mathrm{~kg} / \mathrm{m}^{2}$ ) are both associated with multiple abdominal symptoms leading to reduced QOL. ${ }^{1011}$ Although extensive research has been carried out on the prevalence of obesity in the general population and in connection with numerous 
diseases, there are only little published data on the prevalence and clinical relevance of nutritional status and body mass in patients with IBS. Choung et $a l^{12}$ found no association between IBS status and BMI. However, this study was a population-based study. Studies with large enough sample size based on clinic patients are missing so far.

Therefore, the aim of this study was to describe the BMI distribution in patients with IBS based on the Rome III criteria and to evaluate the association of BMI with symptom severity and the physical health and mental health of QOL.

\section{METHODS}

This cross-sectional survey included patients evaluated at the functional gastrointestinal disorders (FGIDs) specialty clinic of the department of general internal medicine and psychosomatics of Heidelberg University Hospital, which is a tertiary care facility. All patients who completed our routine baseline documentation were enrolled. The routine data from individual health records were transferred into the database and pseudonymised.

\section{Patients recruitment}

From January 2011 to December 2016, patients' clinical data were consecutively collected from our outpatient FGIDs clinic at Heidelberg University Hospital for this explorative and descriptive study. All patients $\geq 18$ years of age, who voluntarily signed an informed consent, were included, if they fulfilled the Rome III criteria for the diagnosis of IBS. ${ }^{1}$ There was no exclusion criteria. The subtype criteria for IBS were based on stool consistency as assessed by the Bristol Stool Scale and Rome III criteria. ${ }^{13}$ Demographic data including gender, age, family status, level of education and residence, were also collected at baseline using the Psychosomatic Basis Documentation Questionnaire. $^{14}$

\section{Patient involvement}

No patients were involved in conducting the study. However, in order to increase the clinical relevance of the study, we established an advisory group to advise the research project.

\section{Measurement of IBS symptom severity}

Patients rated the severity of their IBS symptoms by completing the IBS severity score system (IBS-SSS) ${ }^{15}$ The IBS-SSS has a maximum score of 500 and comprises five items: frequency and intensity of abdominal pain, severity of abdominal distension, dissatisfaction with bowel habits and interference of IBS with daily life. Based on validated cut-off values, three IBS severity subgroups can be distinguished: mild (IBS-SSS: 75-175), moderate (IBSSSS: 175-300) and severe (IBS-SSS:>300). The German version of this questionnaire was validated by Betz et $a l^{16}$, and the total score was computed in accordance with the manual.

\section{Measurement of BMI}

BMI was calculated as the individual's self-reported body weight $(\mathrm{kg})$ divided by the square of their height $(\mathrm{m})$. BMI was categorised according to the WHO classification of physical status ${ }^{17}$ : underweight (BMI $<18.5 \mathrm{~kg} / \mathrm{m}^{2}$ ), normal weight (BMI $\left.18.5-25 \mathrm{~kg} / \mathrm{m}^{2}\right)$, overweight $\left(25-30 \mathrm{~kg} / \mathrm{m}^{2}\right)$ or obese body condition (BMI $\left.>30 \mathrm{~kg} / \mathrm{m}^{2}\right)$.

\section{Measurement of QOL}

QOL was measured using the Short Form 36 Health Survey (SF-36). ${ }^{18}$ SF-36 is a 36 -item, patient-reported survey of patient QOL, which consists of a physical and mental health indexes. The SF-36 is widely used and well validated for assessing generic health outcomes. Each scale is directly transformed into a 0-100 scale. Lower scores represent a higher degree of disability. Validation of the German version was performed by Morfeld et al. ${ }^{19}$

\section{Statistical analysis}

Routine data were transformed into an SPSS file and evaluated using the statistical program SPSS (IBM, V.22.0). Descriptive statistics are presented as the means and SD for continuous variables and as absolute numbers and percentages for categorical variables. All analyses were explorative and not of a confirmatory nature. All primary and secondary variables were first tested for normal distribution. For normally distributed variables, the mean and SD were calculated. Variables that lacked a normal distribution were reported using the median and IQR. The Mann-Whitney U Test was used to assess the level of significance because a number of participants differed between the groups. Comparison of sociodemographic and anthropometric data according to BMI was performed using analysis of variance (ANOVA) and non-parametric tests in the first instance. Where significant group differences were detected based on ANOVA, post-hoc least significant difference tests were conducted to compare the study groups in a pairwise fashion. We also used the Pearson correlation coefficients to investigate the linear association between demographics, BMI, clinical and psychological features. Hierarchical multiple regression was used to help understand the impact of potential confounders as well as the predictive role of BMI in physical health and mental health of QOL. We tested the impact of the confounders (gender, age, family status, education status and IBS subtypes) in model 1. In model 2, we added the symptom severity. BMI categories (underweight, normal weight, overweight and obesity) were added in model 3. All tests were two-sided, and statistical significance was accepted if $\mathrm{p}<0.05$.

\section{RESULTS}

\section{Characteristics of study sample}

A total of 576 patients completed the questionnaire. Excluding 113 patients whose Rome III criteria data were partly missing, 366 patients with IBS were enrolled in this study (114 men, 252 women; mean BMI $23.90 \pm 5.22 \mathrm{~kg} /$ 
Table 1 Distribution of BMl at symptom severity and QOL based on several demographic and clinical features $(n=366)$

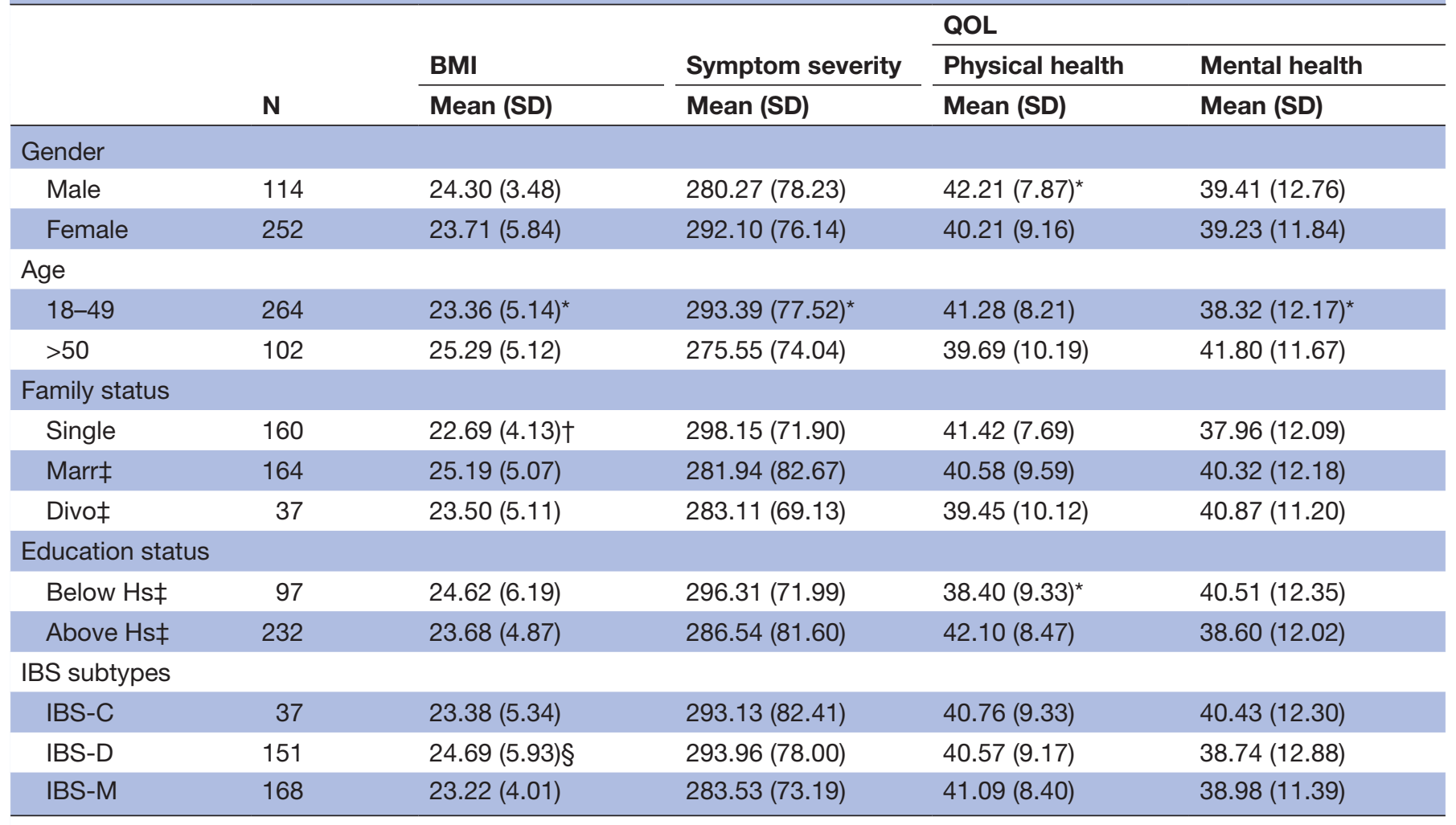

IBS- $U$ was not included in the analysis because the sample size is too small.

Symptom severity based on IBS-SSS: mild (IBS-SSS: 75-175), moderate (IBS-SSS: 175-300) and severe (IBS-SSS: >300).

QOL based on SF-36: range from 0 ('highest disability') to 100 ('no disability').

${ }^{*} \mathrm{P}<0.05$.

$\dagger \mathrm{P}<0.05$ for post-hoc comparison between single and married or unmarried cohabitation.

$\ddagger$ Marr, married or unmarried cohabitation; Divo, divorced or widowed; Hs, high school.

$\S \mathrm{P}<0.05$ for post-hoc comparison between IBS-D and IBS-M.

BMI, body mass index; IBS-C, irritable bowel syndrome (IBS) with constipation; IBS-D, IBS with diarrhoea; IBS-M, mixed IBS; IBS-SSS,

severity score system; QOL, quality of life.

$\mathrm{m}^{2}$ ). The demographic and baseline characteristics of the patients with IBS among the different subtypes are summarised in table 1 . Of the included subjects, the most frequent IBS subtype was IBS-M $(45.9 \%)$, followed by IBS-D $(41.3 \%)$ and IBS-C (10.1\%). Only $59.0 \%$ of patients with IBS were within the normal weight range, whereas $30.3 \%$ were overweight or obese and only a minority were underweight $(10.7 \%)$. The percentage of obese patients was highest in IBS-D $(17.9 \%, \mathrm{p}<0.01)$. Taking gender into account, $5.3 \%$ of men and $13.1 \%$ of women were underweight. Additionally, $7.0 \%$ of men and $13.1 \%$ of women were obese, and $26.3 \%$ of men and $15.9 \%$ of women were overweight. Overall, patients reported moderate to severe IBS symptom severity (IBS-SSS range: 125-484) and lower QOL (physical health range of SF-36: 15.19-64.00, mental health range of SF-36: 7.89-65.85).

\section{Correlations between BMI, symptom severity and Q0L}

Pearson product-moment correlations indicated that high BMI values and elevated symptom severity were associated with poorer QOL. As shown in table 2, BMI was negatively correlated with physical health $(\mathrm{r}=-0.177, \mathrm{p}<0.01)$.

Table 2 Correlation matrix for the study variables in patients with irritable bowel syndrome

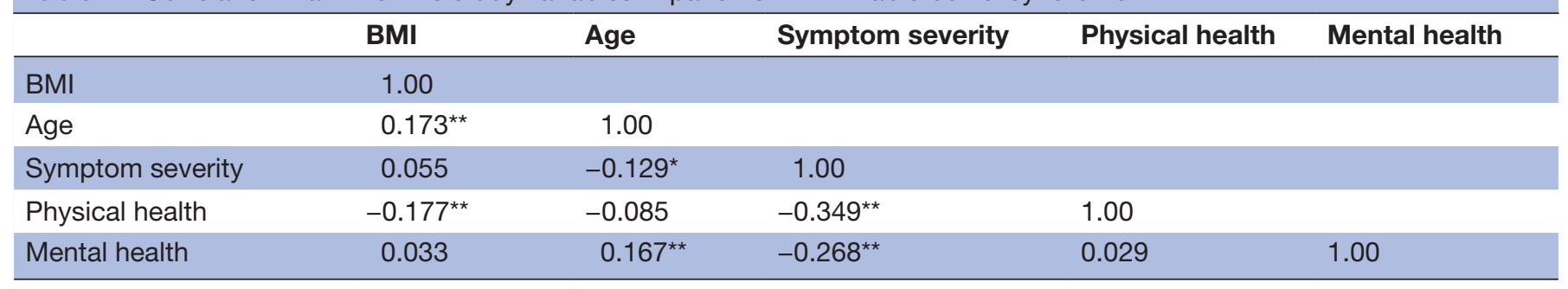

${ }^{*} \mathrm{P}<0.05 ;{ }^{* *} \mathrm{P}<0.01$.

BMI, body mass index. 
Symptom severity was negatively correlated with physical health $(\mathrm{r}=-0.394, \mathrm{p}<0.01)$ and mental health $(\mathrm{r}=-0.268$, $\mathrm{p}<0.01)$. Additionally, age was negatively correlated with symptom severity $(\mathrm{r}=-0.129, \mathrm{p}<0.05)$ and positively correlated with physical health $(\mathrm{r}=0.167, \mathrm{p}<0.01)$.

\section{Characteristics of BMI, symptom severity and Q0L across demographic and IBS subtypes}

As shown in table 1, male reported significantly higher physical health problems than female $(\mathrm{t}=2.141, \mathrm{p}<0.05)$. Compared with older patients (age $>50$ ), the younger patients (age 18-49) reported significantly lower BMI level ( $\mathrm{t}=-3.20, \mathrm{p}<0.01)$, greater symptom severity $(\mathrm{t}=2.039$, $\mathrm{p}<0.05)$ and worse mental health $(\mathrm{t}=-2.528, \mathrm{p}<0.05)$. Patients living with a stable partner (married or unmarried cohabitation) had higher BMI compared with single participants $(\mathrm{t}=-4.397, \mathrm{p}<0.01)$. The patients with below high school education reported significantly higher physical health problems than those who with above high school education $(\mathrm{t}=-3.376, \mathrm{p}<0.01)$. Compared with patients with IBS-M, the patients with IBS-D reported significantly higher BMI level $(\mathrm{t}=-2.572, \mathrm{p}<0.05)$.

\section{The influences of BMI in physical health and mental health of QOL}

As shown in table 3, the hierarchical multiple regression examined the relationship between dependent variable (physical health) and independent variables (symptom severity and BMI). Model 1 was significant $(\mathrm{F}=2.252$, $\mathrm{p}<0.05)$ and explained $5.2 \%$ of the variance in physical health. Education status $(\beta=0.174, p<0.01)$ was a significant positive factor that influencing physical health. In model 2, symptom severity significantly added to the amount of explained variance $\left(\Delta \mathrm{R}^{2}=0.147, \triangle \mathrm{F}=52.498\right.$, $\mathrm{p}<0.01)$. In model $3, \mathrm{BMI}$ also significantly added to the amount of explained variance $\left(\Delta \mathrm{R}^{2}=0.037, \Delta \mathrm{F}=4.605\right.$, $\mathrm{p}<0.01)$. Obesity $(\beta=-0.177, \mathrm{p}<0.01)$ was a significant negative factor that influencing physical health. Similarly, when mental health was used as the dependent variable, model 1 was insignificant $(\mathrm{F}=0.854, \mathrm{p}>0.05)$. In model 2 , symptom severity significantly increased the amount of explained variance $\left(\triangle \mathrm{R}^{2}=0.084, \Delta \mathrm{F}=26.824, \mathrm{p}<0.01\right)$. In model 3, BMI didn't account for an additional significant amount of variance in mental health $\left(\Delta R^{2}=0.007\right.$, $\triangle \mathrm{F}=0.772, \mathrm{p}>0.05)$.

\section{DISCUSSION}

This study sought to describe the BMI distribution in patients with IBS based on the Rome III criteria and to evaluate the association of BMI with symptom severity and the physical health and mental health of QOL. Therefore, we used standardised questionnaires and medical records to confirm the diagnosis and to assess patient symptoms and QOL.

Our data show that being overweight is a common phenomenon in patients with IBS regardless of IBS subtype. These findings are in agreement with the results of a previous study based on obese patients in France, which found that $30.0 \%$ of obese patients had IBS. ${ }^{20} \mathrm{We}$ found that almost $40 \%$ of patients with IBS were not in the normal weight range, which is consistent with previous results. ${ }^{21}$ Notably, the overweight and obesity rates in the general adult population in Germany ${ }^{7}$ are higher than those found in our IBS cohort. According to the German Health Update, ${ }^{7}$ in 2012 , the overweight rate of adults was $36.2 \%$, and the obesity rate was $16.5 \%$. Interestingly, the distribution of weight was similar between the different IBS groups. In particular, there was no difference between IBS-C and IBS-M. Notably, in IBS-D, the percentage of obese patients reached $17.9 \%$. Lee et a $t^{22}$ evaluated the relationship between visceral adipose tissue and the risk of IBS and suggested that disturbances of visceral fat may be more common in patients with IBS-D. In addition, another Korean study found increased intestinal permeability in patients with IBS-D. ${ }^{23}$ One possible explanation is that the increase in visceral fat leads to an increase in intestinal osmolality ${ }^{24}$ and then leads to the chronic diarrhoea. These studies, however, were confined to patients from South Korea.

IBS has a significant impact on patients' QOL. ${ }^{25}$ Our data show an association between symptom severity and QOL with regard to physical and mental health. The patients in our study reported moderate to severe IBS symptom severity and lower QOL. Further, this association followed a negative dose-response pattern. Our findings partially match those of Amouretti et al, ${ }^{26}$ who found that patients with IBS who reported their symptoms as severe or very severe had a very poor QOL compared with those who reported their symptoms as moderate. However, their study did not distinguish between physical and mental health and did not consider the effects of confounding factors such as BMI. This is an interesting but not completely new result which gives rise to the question of what mechanisms are responsible for this association.

Through the hierarchical multiple regression, we tested the relationship between dependent variable (QOL) and independent variables (symptom severity and BMI). We controlled for the impact of the confounders (gender, age, family status, education status and IBS subtypes). The correlation between symptom severity and QOL was significantly negative, no matter which BMI category was. Our findings show that obesity was significant negative predictor of physical health. Patients with higher BMI were more frequently in poor physical health. This is in line with previous studies, ${ }^{11}$ in that increasing BMI is associated with increased upper gastrointestinal symptoms, bloating and diarrhoea. Obesity may lead to more physiological stress on organs. Richards $e t a l^{27}$ reported that obese patients have more severe pain and are to a larger extent restricted in their daily functioning compared with patients of normal weight. An additional finding of our study was that BMI didn't account for an additional significant amount of variance in mental health. This was inconsistent with findings by Mykletun $e t a l,{ }^{28}$ who found 


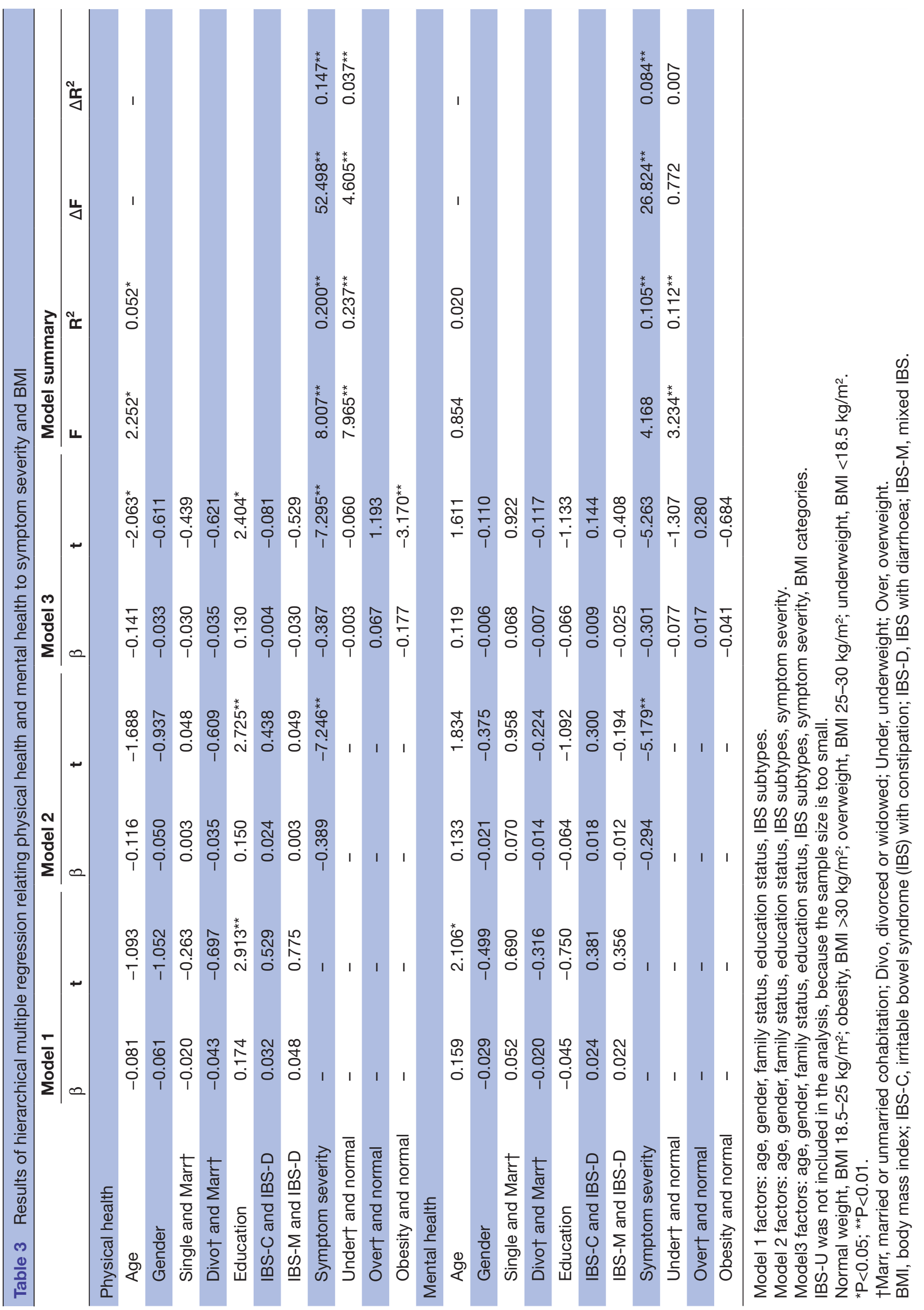


that BMI had significant association with IBS with regard to anxiety and mood disorders. However, their study evaluated only female patients. The findings of genome-wide association studies, from the genetic perspective, suggest the presence of many genetic loci each with a small effect influencing susceptibility to mental health symptoms (depression and anxiety). ${ }^{29}$ We can therefore hypothesise that, the unpredictable association between risk of mental health and BMI in our study may be due to non-modifiable genetic influences which predispose individuals to bad mental health.

Several limitations of this study must be taken into account. First, the study was cross-sectional, therefore, it is impossible to infer causation. Second, BMI was based on the self-reported height and weight of patients and computed without objective measurement, therefore bias may have been introduced. Patients may occasionally under-report or over-report their weight and height leading to an underestimation of underweight or obese patients. However, in the Nutrinet-Santé study, researchers reported that deviations in self-reported BMIs from questionnaires can be ignored because their results confirmed the validity and agreement of self-reported data with measured data. ${ }^{3031}$ The choice of SF-36 as the only QOL tool may partly miss the relationship between symptom severity and QOL. Moreover, BMI is associated with a multitude of different factors, such as genetics, fatty mass, dietary habit or physical exercise. Thus, BMI cannot fully reflect the impact of those factors on IBS in more details, and more research is needed. The strengths of this study, however, are the use of a large patient cohort with a validated diagnosis of IBS based on the Rome III criteria.

To conclude, being overweight is a common phenomenon in patients with IBS regardless of IBS subtypes. Our data further suggest that overweight and obesity may have a relevant influence on QOL. Patients with higher BMI were more frequently in poor physical health. The findings have some implications for future practice. Clinical doctors should pay special attention to abnormal weight in patients with IBS as this maybe an indicator of a poorer QOL, especially with regard to the physical health.

Contributors YD, JT, RS, WE: designed the study and wrote the protocol. YD, SB, JT: performed the literature searches and analyses. YD: wrote the first draft of the manuscript. All of the authors contributed to and have approved the final manuscript.

Funding The authors have not declared a specific grant for this research from any funding agency in the public, commercial or not-for-profit sectors.

Competing interests None declared.

Patient consent Obtained.

Ethics approval This study was approved by the Ethics Committee of Heidelberg University (S-041/2014) and carried out in accordance with the Declaration of Helsinki.

Provenance and peer review Not commissioned; externally peer reviewed. Data sharing statement There is no additional unpublished data from this study. Open access This is an open access article distributed in accordance with the Creative Commons Attribution Non Commercial (CC BY-NC 4.0) license, which permits others to distribute, remix, adapt, build upon this work non-commercially, and license their derivative works on different terms, provided the original work is properly cited, appropriate credit is given, any changes made indicated, and the use is non-commercial. See: http://creativecommons.org/licenses/by-nc/4.0/.

\section{REFERENCES}

1. Drossman DA. The functional gastrointestinal disorders and the Rome III process. Gastroenterology 2006;130:1377-90.

2. Lacy BE. Perspective: an easier diagnosis. Nature 2016;533:S107.

3. Ljótsson B, Hesser H, Andersson E, et al. Provoking symptoms to relieve symptoms: a randomized controlled dismantling study of exposure therapy in irritable bowel syndrome. Behav Res Ther 2014;55:27-39.

4. Hoekman DR, Rutten JM, Vlieger AM, et al. Annual costs of care for pediatric irritable bowel syndrome, functional abdominal pain, and functional abdominal pain syndrome. J Pediatr 2015;167:1103-8.

5. Hayes $\mathrm{P}$, Corish $\mathrm{C}, \mathrm{O}^{\prime}$ Mahony $\mathrm{E}$, et al. A dietary survey of patients with irritable bowel syndrome. J Hum Nutr Diet 2014;27(Suppl 2):36-47.

6. Eswaran S, Chey WD, Jackson K, et al. A diet low in fermentable oligo-, di-, and monosaccharides and polyols improves quality of life and reduces activity impairment in patients with irritable bowel syndrome and diarrhea. Clin Gastroenterol Hepatol 2017;15:1890-9.

7. Lange C, Jentsch F, Allen J, et al. Data resource profile: German Health Update (GEDA)-the health interview survey for adults in Germany. Int J Epidemiol 2015;44:442-50.

8. Gracey M, King M. Indigenous health part 1: determinants and disease patterns. Lancet 2009;374:65-75.

9. Rizzi M, Mazzuoli S, Regano N, et al. Undernutrition, risk of malnutrition and obesity in gastroenterological patients: a multicenter study. World J Gastrointest Oncol 2016;8:563.

10. van Oijen MG, Josemanders DF, Laheij RJ, et al. Gastrointestinal disorders and symptoms: does body mass index matter? Neth $\mathrm{J}$ Med 2006;64:45-9.

11. Talley NJ, Howell S, Poulton R. Obesity and chronic gastrointestinal tract symptoms in young adults: a birth cohort study. Am J Gastroenterol 2004;99:1807-14.

12. Choung RS, Locke GR, Zinsmeister AR, et al. Psychosocial distress and somatic symptoms in community subjects with irritable bowel syndrome: a psychological component is the rule. Am J Gastroenterol 2009;104:1772-9.

13. Heaton KW, Gosh S. Relation between stool from on a seven point scale and symptoms of urgency, straining, and incomplete evacuation. Gut 1989;30:A1465.

14. Heuft G. Praxis der Qualitätssicherung in der Psychotherapie: das Manual zur Psy-BaDo; [entsprechend den Empfehlungen der psychotherapeutischen Fachgesellschaften]. Germany: Thieme, 1998.

15. Kaptchuk TJ, Friedlander E, Kelley JM, et al. Placebos without deception: a randomized controlled trial in irritable bowel syndrome. PLoS One 2010;5:e15591.

16. Betz C, Mannsdörfer K, Bischoff SC. [Validation of the IBS-SSS]. Z Gastroenterol 2013;51:1171-6.

17. World Health Organization. Physical status: The use of and interpretation of anthropometry, Report of a WHO Expert Committee. Geneva: World Health Organization, 1995.

18. Jenkinson C, Coulter A, Wright L. Short form 36 (SF36) health survey questionnaire: normative data for adults of working age. BMJ 1993;306:1437-40.

19. Morfeld M, Kirchberger I, Bullinger M. SF-36 Fragebogen zum Gesundheitszustand: Deutsche Version des Short Form-36 Health Survey: Hogrefe, 2011.

20. Schneck AS, Anty R, Tran A, et al. Increased prevalence of irritable bowel syndrome in a cohort of French morbidly obese patients candidate for bariatric surgery. Obes Surg 2016;26:1525-30.

21. Ruigómez A, Wallander MA, Johansson S, et al. One-year followup of newly diagnosed irritable bowel syndrome patients. Aliment Pharmacol Ther 1999;13:1097-102.

22. Lee CG, Lee JK, Kang YS, et al. Visceral abdominal obesity is associated with an increased risk of irritable bowel syndrome. Am J Gastroenterol 2015;110:310-9.

23. Lee H, Park JH, Park DI, et al. Mucosal mast cell count is associated with intestinal permeability in patients with diarrhea predominant irritable bowel syndrome. J Neurogastroenterol Motil 2013;19:244-50.

24. Eslick GD. Gastrointestinal symptoms and obesity: a meta-analysis. Obes Rev 2012;13:469-79.

25. Neri L, lovino P. Laxative Inadequate Relief Survey (LIRS) Group. Bloating is associated with worse quality of life, treatment satisfaction, and treatment responsiveness among patients with 
constipation-predominant irritable bowel syndrome and functional constipation. Neurogastroenterol Motil 2016;28:581-91.

26. Amouretti M, Le Pen C, Gaudin AF, et al. Impact of irritable bowel syndrome (IBS) on health-related quality of life (HRQOL). Gastroenterol Clin Biol 2006;30:241-6.

27. Richards MM, Adams TD, Hunt SC. Functional status and emotional well-being, dietary intake, and physical activity of severely obese subjects. J Am Diet Assoc 2000;100:67-75.

28. Mykletun A, Jacka F, Williams $L$, et al. Prevalence of mood and anxiety disorder in self reported irritable bowel syndrome (IBS).
An epidemiological population based study of women. BMC Gastroenterol 2010;10:88

29. Demirkan A, Penninx BW, Hek K, et al. Genetic risk profiles for depression and anxiety in adult and elderly cohorts. Mol Psychiatry 2011;16:773-83.

30. Lassale C, Péneau S, Touvier M, et al. Validity of web-based selfreported weight and height: results of the Nutrinet-Santé study. $J$ Med Internet Res 2013;15:e152.

31. Siah KT, Wong RK, Chan YH, et al. Prevalence of irritable bowel syndrome in singapore and its association with dietary, lifestyle, and environmental factors. J Neurogastroenterol Motil 2016;22:670-6. 